\title{
Utilização dos protocolos clínicos e diretrizes terapêuticas por médicos da atenção básica
}

\author{
Use of Clinical Protocols and Therapeutic Guidelines by \\ Basic Health Care physicians
}

Ana Lúcia Couto Coronel', Lisiane Marcolin de Almeida',

Rita Catalina Aquino Caregnato', Helena Terezinha Hubert Silva'

'Programa de Pós-graduação Ensino na Saúde, Universidade Federal de Ciências da Saúde,

Porto Alegre (RS), Brasil.

\section{RESUMO}

Objetivo: identificar o conhecimento e utilização dos Protocolos Clínicos e Diretrizes Terapêuticas (PCDT) pelos médicos da Atenção Básica. Método: estudo exploratório descritivo, por meio de questionários online enviados aos médicos que trabalham na Atenção Básica em Porto Alegre, RS. Os questionários foram enviados por correio eletrônico às coordenações das Unidades Básicas de Saúde do município, para que fossem entregues médicos que atuam na área. Resultados: somente 31 médicos responderam $(5,71 \%)$, sendo que 27 (87,1\%) afirmaram conhecer os PCDT, mas somente 16 (51,6\%) os utilizam na prática diária. O principal motivo alegado para a não utilização foi "falta de tempo". Conclusão: os dados sugerem que os médicos da Atenção Básica em Porto Alegre conhecem os PCDT, mas poucos os utilizam no dia a dia. Portanto, novos estudos devem avaliar as causas disso e incentivar a utilização dos PCDT para o desenvolvimento de melhores práticas.

Palavras-chave: Medicina baseada em evidências. Protocolos clínicos. Assistência integral à saúde.

\begin{abstract}
Objective: To identify the knowledge and use of Clinical Protocols and Therapeutic Guidelines (PCDT) by primary care doctors. Method: descriptive exploratory study, through online questionnaires sent to the doctors who work in Primary Care in Porto Alegre, RS. The questionnaires were sent by e-mail to the coordinators of the Basic Health Units of the city, thus that doctors who work in the area could be delivered. Results: Only 31 doctors answered (5.71\%), and 27 (87.1\%) reported knowing the PCDTs, but only 16 (51.6\%) used them in daily practice. The main reason alleged for non-use was "lack of time". Conclusion: the data suggest that Basic Care doctors in Porto Alegre are familiar with PCDT, but only few use it on a daily. Therefore, further studies should assess the causes of this and encourage the use of PCDTs for the development of best practices.
\end{abstract}

Keywords: Evidence-based medicine. Clinical protocols. Integral health care.

Recebido: Jun. 15, 2017 Aceito: Jan. 08, 2018

\section{COMO CITAR ESTE ARTIGO}

Coronel ALC, Almeida LM Caregnato RCA, Silva HTH. Utilização dos protocolos clínicos e diretrizes terapêuticas por médicos da atencão básica. Interdisciplinary Journal of Health Education. 2017 Jul-Dez;2(2):96-102. https://doi.org/10.4322/ijhe.2017.004

\section{CORRESPONDÊNCIA}

Ana Lúcia Couto Coronel

Programa de Pós-graduação Ensino

na Saúde, Universidade Federal de

Ciências da Saúde

Rua Sarmento Leite, 245

CEP 90050-170, Porto Alegre (RS),

Brasil

Tel.: (51) 3303-9000

alc.coronel@hotmail.com

\section{CONFLITO DE INTERESSE}

Os autores declararam não

haver conflitos de interesse.

O estudo foi realizado nas Unidades Básicas de Saúde e Unidades de Saúde da Família, Porto Alegre (RS), Brasil.

Todos os autores leram e aprovam a versão final submetida ao Interdisciplinary Journal of Health Education (IJHE). 


\section{Introdução}

A medicina é uma área direcionada principalmente à aplicação do conhecimento e os critérios utilizados por cada profissional na sua prática são amplamente variáveis, mesclando intuição, conhecimento adquirido, pragmatismo, experiência prévia, informações relevantes e confiabilidade de fontes consultadas. Contudo, esse conjunto de informações nem sempre garante a melhor escolha na hora da tomada de decisão ${ }^{1}$.

Medicina Baseada em Evidências (MBE) é um conceito que vem sendo amplamente adotado, com o objetivo de dar maior segurança e confiabilidade às tomadas de decisão na prática médica ${ }^{2}$. Consiste na utilização das melhores evidências externas disponíveis, advindas de pesquisas sistemáticas, para sustentar diagnósticos e condutas terapêuticas ${ }^{3}$. Os princípios básicos da MBE são: formulação de uma questão clara relacionada ao problema do paciente, pesquisa na literatura de informações relevantes para essa questão, avaliação crítica do valor e da aplicabilidade dessas informações e da implementação do seu uso na prática clínica ${ }^{2}$. Para esse exercício é necessário tempo, acesso à literatura disponível, metodologia e condições para aplicar as diretrizes coletadas ${ }^{4}$, sendo que as evidências assumem real valor quando aliadas à experiência profissional e à personalização das informações, relacionadas ao indivíduo a quem serão direcionadas ${ }^{5}$.

A atividade científica pressupõe a produção, circulação e incorporação do conhecimento $^{6}$. O aumento da produção científica e a demanda crescente nos serviços de saúde são fatores que limitam os profissionais no cumprimento dos passos necessários para embasar a prática clínica, a partir das melhores evidências disponíveis. Atendendo a essas dificuldades, as pesquisas sistemáticas vêm sendo desenvolvidas pelos pesquisadores, pois resumem o conhecimento adquirido em diversas áreas, a partir de pesquisas realizadas com rigor metodológico, definindo as melhores diretrizes a serem adotadas ${ }^{7}$. A categorização das evidências encontradas e classificação quanto ao seu nível e grau de recomendação, também surgiram para conferir maior confiabilidade e facilitar o desenvolvimento de protocolos que auxiliam nas tomadas das decisões em saúde ${ }^{8,9}$.

No Brasil, a partir de 2000, visando incorporar a prática baseada em evidências ao Sistema Único de Saúde (SUS) e a melhor utilização das novas tecnologias, o Ministério da Saúde (MS) implementou a criação de Protocolos Clínicos e Diretrizes Terapêuticas (PCDT). Essa implementação foi normatizada em 2011 pela Lei n ${ }^{\circ} 12.401$, tendo passado por alguns processos de aprimoramento desde o seu início ${ }^{10}$. Consultas públicas e avaliações da Comissão Nacional de Incorporação de Tecnologias no SUS (CONITEC) têm servido de bases para a formulação dos PCDT, os quais visam estabelecer os critérios de diagnóstico de cada doença, a sequência de escolhas para o tratamento das doenças e os meios adequados para monitorar a efetividade e efeitos adversos desses tratamentos ${ }^{11,12}$. Os PCDT têm sido atualizados desde a sua criação e as últimas edições procuram seguir critérios internacionais para a elaboração de protocolos ${ }^{13,14}$.

Sem ignorar aspectos controversos da prática baseada em evidências, que vão desde possíveis manipulações comerciais até falsas rupturas paradigmáticas ${ }^{15}$, é importante reconhecer a necessidade de incrementar o arsenal de recursos disponíveis aos médicos que atuam no SUS, assim como orientar a melhor utilização desses recursos. O SUS, que tem como um dos seus princípios a assistência integral à saúde, apresenta diferentes níveis de complexidade, desde a atenção básica até o nível hospitalar, com consumo variável das tecnologias em saúde ${ }^{16,17}$. Tendo em vista que a atenção básica é a "porta de entrada" para o SUS, nela as tomadas de decisão têm maior impacto no consumo das tecnologias ${ }^{18}$. A partir disso, esta pesquisa teve como objetivo avaliar o 
conhecimento e a utilização dos Protocolos Clínicos e Diretrizes Terapêuticas (PCDT) criados pelo Ministério da Saúde, pelos médicos da Atenção Básica do município de Porto Alegre, RS.

\section{Métodos}

Este é um estudo exploratório descritivo proposto pela Universidade Federal de Ciências da Saúde (UFCSPA), através do Programa de Mestrado Profissional Ensino na Saúde, em parceria com a Secretaria Municipal de Saúde (SMS) de Porto Alegre, tendo sido aprovado pelas Comissões de Ética em Pesquisa de ambas as instituições, sob os registros CAAE 58362016.7.0000.5345 e CAAE 58362016.7.3001.5338. A coleta de dados ocorreu de dezembro de 2016 a março de 2017.

O público-alvo da pesquisa foi constituído pelos médicos das Unidades Básicas de Saúde (UBS) e Unidades de Saúde da Família (USF) do município de Porto Alegre, RS. Como critério de inclusão foi adotado: ser médico atuante na assistência de uma UBS ou USF. Segundo informações da Secretaria Municipal de Saúde de Porto Alegre, durante o período de coleta de dados haviam 543 médicos atuantes na Atenção Básica do município. Para essa população, o cálculo amostral para um Intervalo de confiança de $95 \%$ e uma margem de erro de $5 \%$ foi de 226 participantes.

O instrumento utilizado para a coleta de dados foi um questionário semiestruturado, elaborado na ferramenta Google Docs. Inicialmente, uma carta-convite, contendo o link para o Termo de Consentimento Livre e Esclarecido (TCLE) e para o questionário, foi encaminhada por correio eletrônico às coordenações das 55 Unidades Básicas de Saúde (UBS) e 88 Unidades de Saúde da Família (USF) do município, para ser repassada aos médicos das referidas unidades. Os endereços eletrônicos institucionais dos coordenadores foram fornecidos pela Coordenação Geral da Atenção Básica, que referiu não poder divulgar os endereços eletrônicos dos médicos. O questionário apresentava oito perguntas: idade, tipo de unidade em que trabalha (UBS/USF), área de atuação, se tem conhecimento dos PCDT e como conheceu (mídia impressa/eletrônica), se utiliza os PCDT na prática diária, quais os protocolos que costuma utilizar e, se não utiliza, qual o motivo. Após o envio para as coordenações, foi realizado contato com as Gerências Distritais e com a Coordenação Geral da Atenção Básica, a fim de promover a divulgação e importância da pesquisa. Foram realizados três novos contatos por correio eletrônico com as coordenações das unidades e um com as Gerências Distritais da Atenção Básica de Porto Alegre, a fim de reforçar a importância do estudo e estimular a adesão. Contatos telefônicos também foram realizados com as unidades onde não houve adesão dos médicos.

Os dados coletados foram revisados e digitados no software Epidata 3.0 (Epidata Association, Odense, Dinamarca) e submetidos à análise descritiva de frequências absolutas e relativas.

\section{Resultados}

Do total de 543 médicos atuantes na atenção básica de Porto Alegre no momento da coleta de dados, somente 31 médicos $(5,71 \%)$ responderam o questionário. Sendo assim, o tamanho reduzido da amostra limitou a aplicação de testes estatísticos e a generalização dos resultados. Por esse motivo, foram realizadas apenas distribuições de frequências absolutas e relativas e a análise descritiva dos dados. Apesar dessa limitação, a análise descritiva dos dados possibilita a reflexão crítica sobre o perfil encontrado e estimula a discussão sobre a prática baseada em evidências e a utilização 
de recursos disponibilizados pelo Ministério da Saúde aos médicos da atenção básica, apontando para uma importante lacuna a ser investigada.

Na Tabela 1 são apresentados os dados relativos ao perfil dos profissionais desta amostra. Entre os 31 respondentes da pesquisa, dezesseis $(51,6 \%)$ tinham mais de 40 anos, cinco $(16,13 \%)$ tinham entre 35 e 40 anos, seis $(19,35 \%)$ tinham entre 31 e 35 anos e quatro (12,9\%) tinham entre 26 e 30 anos de idade. Na distribuição por especialidade, 23 são médicos de família, 5 são clínicos gerais, 2 são ginecologistas e 1 profissional respondeu ser de "outra" área. Quanto ao tipo de unidade assistencial, 17 são médicos de UBS e 14 atuam em unidades mistas (USF/UBS).

Vinte e um participantes $(67,7 \%)$ afirmaram conhecer os PCDT, mas somente 14 destes $(66,7 \%)$ referem utilizar os protocolos na prática clínica, sendo que, sobre o total da amostra, esse percentual representa $45,2 \%$. A mídia digital foi o método de acesso aos protocolos para 21 médicos $(77,78 \%)$ e, em ordem de frequência, os cinco protocolos mais utilizados são: Asma, Doenças Sexualmente Transmissíveis e AIDS, Saúde da Mulher, Pré-natal de baixo risco e Tuberculose. Entre os 15 médicos que não utilizam os PCDT, sete $(46,67 \%)$ afirmaram a falta de tempo como motivo para a não utilização, um referiu falta de acesso e os demais alegaram "outros" motivos. A Tabela 2 apresenta a distribuição do conhecimento e utilização dos Protocolos Clínicos e Diretrizes Terapêuticas por área de atuação.

Tabela 1. Perfil profissional, 2017.

\begin{tabular}{lcc}
\multicolumn{1}{c}{ Variáveis } & $\mathbf{N}$ & $\%$ \\
\hline IDADE & 4 & 12,9 \\
\hline $26-30$ anos & 6 & 19,35 \\
\hline $31-35$ anos & 5 & 16,13 \\
\hline $35-40$ anos & 16 & 51,6 \\
\hline$>40$ anos & & \\
\hline ESPECIALIDADE & 23 & 74,19 \\
\hline Médicos de família & 5 & 16,2 \\
\hline Clínicos Gerais & 2 & 6,45 \\
\hline Ginecologistas & 1 & 3,16 \\
\hline Outra área & 17 & 54,8 \\
\hline ATUAÇÃO & 14 & 45,2 \\
\hline UBS & & 100 \\
\hline USF/UBS & & \\
\hline TOTAL &
\end{tabular}

Fonte: Pesquisa direta, elaborada pelos autores, 2017.

Tabela 2. Conhecimento e utilização dos PCDT por área de atuação médica.

\begin{tabular}{ccccc} 
& Conhecem & Não Conhecem & Utilizam & Não Utilizam \\
Área de atuação & $\mathbf{N}$ & $\mathbf{N}$ & $\mathbf{N}$ & $\mathbf{N}$ \\
Médico de família & 21 & 2 & 14 & 4 \\
\hline Clínico geral & 4 & 1 & 1 & 1 \\
\hline Ginecologista & 1 & 1 & - & 1 \\
\hline Outro & 1 & - & 16 & 15
\end{tabular}

Fonte: Pesquisa direta, elaborada pelos autores, 2017. 


\section{Discussão}

A primeira questão a ser discutida e que representa uma limitação deste estudo, foi a escassa participação dos médicos da Atenção Básica de Porto Alegre no estudo proposto. É possível que a metodologia virtual para coleta de dados ainda não tenha o alcance desejado, apesar de vários estudos terem apontado como positiva a utilização da internet e suas ferramentas nas pesquisas em saúde ${ }^{19-22}$. Embora tenham sido respeitadas as normas éticas da pesquisa online, garantindo segurança e sigilo entre pesquisadores e entrevistados, muitos podem ainda se sentir desconfortáveis com essa forma de comunicação ${ }^{23}$. Outra possível razão, apontada por alguns respondentes como motivo para a não utilização dos PCDT, pode ser interpretada como um dos motivos à baixa de adesão: "falta de tempo". Alguns estudos apontam a sobrecarga de trabalho e a difícil gestão do tempo como um dos grandes problemas entre os profissionais da saúde ${ }^{24,25}$. Para essa questão, também é preciso considerar o perfil de comprometimento dos profissionais que atuam no setor estudado, sabendo dos conflitos que frequentemente permeiam as relações de trabalho ${ }^{26}$.

Apesar de representar uma pequena fração do todo, a experiência relatada pelos trinta e um médicos participantes, relacionada ao conhecimento e utilização dos PCDT, alerta para questões importantes a serem consideradas.

Nesse contexto, é necessário considerar que os esforços do Ministério da Saúde (MS) e das equipes envolvidas na elaboração dos PCDT, sem mencionar os custos relacionados ao processo ${ }^{12}$, podem não estar encontrando a repercussão esperada. Estudos com rigor metodológico, considerados o padrão ouro da $\mathrm{MBE}$, visando direcionar a conduta médica por meio de resultados consistentes, são estimulados pela Rede Brasileira de Avaliação de Tecnologias em Saúde (REBRATS). A REBRATS, criada pela Portaria $n^{\circ} 2.915$, de 12 de dezembro de 2011, busca estabelecer a ponte entre pesquisa, política e gestão, fornecendo subsídios para decisões de incorporação, monitoramento e abandono de tecnologias ${ }^{23}$. Os Núcleos de Avaliação de Tecnologias em Saúde (NATS) foram desenvolvidos com o intuito de fortalecer as estratégias da REBRATS nos serviços de saúde, atuando de forma a incentivar e estimular o uso de evidencias científicas nas tomadas de decisões dos profissionais. Embora existam questionamentos relacionados aos métodos de seleção de tecnologias adotados pelo MS e que modelos internacionais sejam sugeridos para esse fim, isso não inviabiliza a melhor utilização dos recursos disponíveis ${ }^{27}$.

Sempre levando em consideração que o conhecimento e a prática são subsídios importantíssimos e insubstituíveis no desempenho profissional, ambos requerem aprimoramento constante ${ }^{28}$ e esse é um dos propósitos da Medicina Baseada em Evidências ${ }^{29}$. O fato de menos da metade dos profissionais investigados relatarem utilizar alguns PCDT na prática clínica, sinaliza para uma possível subutilização de recursos $^{30}$. Em um sistema que enfrenta grandes dificuldades de financiamento e gestão, o desperdício de recursos tem grande impacto na saúde da população e no próprio sistema $^{31}$.

Os PCDT funcionam como uma ferramenta que proporciona estímulo à criação e implementação de políticas e de programas de saúde no SUS, tendo um importante papel na atenção básica, pois é um ambiente onde a fragmentação da saúde está menos presente. Dessa forma, os profissionais devem estar voltados para a corresponsabilidade de saberes clínicos, sociais e comunitários condizentes com a realidade local de cada unidade ${ }^{10}$.

Também merece especial atenção o fato de que metade daqueles que afirmaram não utilizar os PCDT, consideram escasso o tempo disponível para as avaliações e formulações diagnósticas e terapêuticas ${ }^{32}$. É possível especular que, nesse contexto, a demanda é retroalimentada por tomadas de decisão que não contaram com os melhores recursos disponíveis, pois estudos afirmam que o uso dos PCDT proporciona maior qualidade no cuidado prestado ${ }^{28,29}$. Vale ressaltar, que a utilização dos PCDT 
garante a integralidade do cuidado para com os usuários da rede de saúde por meio do monitoramento da efetividade do tratamento. Além disso, os profissionais podem sugerir modificações e/ou revisões para facilitar e estimular o uso desses instrumentos, já que essa utilização exerce importância no controle social quando vinculada a gestão em diferentes níveis de atenção à saúde ${ }^{10}$.

\section{Conclusão}

A partir da amostra estudada, foi possível verificar que a maioria dos médicos da Atenção Básica de Porto Alegre conhece os PCDT. Porém, menos da metade dos respondentes os utiliza como guia na prática assistencial. Esses achados sugerem que as iniciativas do Ministério da Saúde e dos gestores do SUS parecem não estar alcançando completamente seus objetivos, no que se refere à utilização dos PCDT e uso sistematizado de tecnologias.

Novos estudos que avaliem amplamente a utilização dos PCDT por um número maior de usuários e as causas da subutilização desse recurso, poderão direcionar melhores práticas na Assistência Básica.

\section{Referências}

1. Camargo KR Jr, Sobre palheiros, agulhas, doutores e o conhecimento médico: o estilo de pensamento dos clínicos. Cad Saude Publica. 2003;19(4):1163-74. http://dx.doi.org/10.1590/S0102-311X2003000400037. PMid:12973580.

2. Lopes AA. Medicina Baseada em Evidências: a arte de aplicar o conhecimento científico na prática clínica. Rev Assoc Med Bras. 2000;46(3):285-8. http://dx.doi.org/10.1590/S0104-42302000000300015. PMid:11070521.

3. Berwanger O, Guimarães HP, Avezum A. Medicina baseada em evidências. Rev Bras Clín Terap. 2005;31(3):120-6.

4. Rosenberg W, Donald A. Evidence based medicine: an approach to clinical problem-solving. BMJ: British Medical Journal. 1995;310(6987):1122-6. http://dx.doi.org/10.1136/bmj.310.6987.1122. PMid:7742682.

5. Sackett DL, Rosenberg WMC, Gray JAM, Haynes RB, Richardson WS. Evidence based medicine: what it is and what it isn’t. BMJ. 1996;312(7023):71-2. http://dx.doi.org/10.1136/bmj.312.7023.71. PMid:8555924.

6. Pellegrini A Fo. Science for health: notes on the organization of scientific activity for the development of health in Latin America and the Caribbean. Rev Panam Salud Publica. 2000;7(5):345-9. http://dx.doi.org/10.1590/S1020-49892000000500013.

7. Perissé ARS, Gomes MM, Nogueira SA. Revisões sistemáticas (inclusive metanálises) e diretrizes clínicas. In: Gomes MM, organizador. Medicina baseada em evidências: princípios e práticas. Rio de Janeiro (RJ): Reichmann \& Affonso; 2001. p. $131-48$.

8. Centre for Evidence-Based Medicine. Oxford Centre for Evidence-based Medicine - Levels of Evidence (March 2009) [Internet]. Oxford: CEBM; 2009 [citado em 2017 abr 29]. Disponível em: http://www.cebm.net/ oxford-centre-evidence-based-medicine-levels-evidence-march-2009/

9. Moher D, Liberati A, Tetzlaff J, Altman DG. Preferred reporting items for systematic reviews and meta-analyses: the PRISMA statement. Ann Intern Med. 2009;151(4):264-9, W64. http://dx.doi.org/10.7326/0003-4819-151-4-20090818000135. PMid:19622511.

10. Mega TP, Lopes ACDF, Santos VCC, Petramale CA. Protocolos clínicos e diretrizes terapêuticas no SUS: histórico, desafios e perspectivas. Gestão e Saúde. 2015;6(4):3275-85. http://dx.doi.org/10.18673/gs.v6i4.22111.

11. CONITEC: Comissão Nacional de Incorporação de Tecnologias no SUS [Internet]. Protocolos e diretrizes. Brasília: CONITEC; 2016 [citado em 2018 Maio 23]. Disponível em: http://conitec.gov.br/protocolos-e-diretrizes

12. Brasil. Ministério da Saúde. Secretaria de Ciência, Tecnologia e Insumos Estratégicos. Departamento de Ciência e Tecnologia. Nova Comissão Nacional de Incorporação de Tecnologias de Saúde e impacto ao Sistema Único de Saúde. Rev Saúde Pública. 2011;45(5):993-6. http://dx.doi.org/10.1590/S0034-89102011000500024. PMid:21986712.

13. Terrace L. Development and validation of an international appraisal instrument for assessing the quality of clinical practice guidelines: the AGREE project. Qual Saf Health Care. 2003;12(1):18-23. http://dx.doi.org/10.1136/qhc.12.1.18. PMid:12571340.

14. Ronsoni RM, Pereira CC, Stein AT, Osanai MH, Machado CJ. Avaliação de oito Protocolos Clínicos e Diretrizes Terapêuticas (PCDT) do Ministério da Saúde por meio do instrumento AGREE II: um estudo piloto. Cad Saude Publica. 2015;31(6):115762. http://dx.doi.org/10.1590/0102-311X00118814. PMid:26200364.

15. Vasconcellos-Silva PR, Castiel LD. Proliferação das rupturas paradigmáticas: o caso da medicina baseada em evidências. Rev Saude Publica. 2005;39(3):498-506. http://dx.doi.org/10.1590/S0034-89102005000300024. PMid:15997329.

16. Paim J. O que é o SUS: SciELO. Rio de Janeiro: Editora FIOCRUZ; 2009.

17. Coronel ALC, Bonamigo AW, Azambuja MS, Silva HTH. Sistema Único de Saúde (SUS): quando vai começar? IJHEInterdisciplinary Journal of Health Education. 2017;1(2):83-90.

18. Giovanella L, Escorel S, Mendonça MHM. Porta de entrada pela atenção básica? Integração do PSF à rede de serviços de saúde. Saúde Debate. 2003;27(65):278-89.

19. Castro RCF. Impacto da Internet no fluxo da comunicação científica em saúde. Rev Saude Publica. 2006;40:57-63. 


\section{ighe}

20. Vivancos R, Schelenz S, Loke YK. Internet treatment of sexually transmitted infections-a public health hazard? BMC Public Health. 2007;7(1):333. http://dx.doi.org/10.1186/1471-2458-7-333. PMid:18005431.

21. Scotch M, Yip KY, Cheung K-H. Development of grid-like applications for public health using Web 2.0 mashup techniques. J Am Med Inform Assoc. 2008;15(6):783-6. http://dx.doi.org/10.1197/jamia.M2731. PMid:18755998.

22. Gao S, Mioc D, Yi X, Anton F, Oldfield E, Coleman DJ. Towards web-based representation and processing of health information. Int J Health Geogr. 2009;8(1):3. http://dx.doi.org/10.1186/1476-072X-8-3. PMid:19159445.

23. Honorato EJSA. A interface entre saúde pública e cibercultura. Cien Saude Colet. 2014;19(2):481-5. http://dx.doi. org/10.1590/1413-81232014192.21512012. PMid:24863825.

24. Barros NMGC, Honório LC. Riscos de adoecimento no trabalho de médicos e enfermeiros em um hospital regional matogrossense. REGE-Revista de Gestão. 2015;22(1):21-39. http://dx.doi.org/10.5700/rege549.

25. Tironi MOS, Teles JMM, Barros DS, et al. Prevalência de síndrome de burnout em médicos intensivistas de cinco capitais brasileiras. Rev Bras Ter Intensiva. 2016;28(3):270-7. PMid:27737426.

26. Sanches EN, Próspero ENS, Stuker H, Borba MC Jr. Organização e trabalho: padrões de comprometimento dos profissionais que atuam na estratégia saúde da família. Revista Eletrônica de Enfermagem. 2010;12(2):294-300.

27. Soarez PC, Novaes HMD. Limiares de custo-efetividade e o Sistema Único de Saúde. Cad Saude Publica. 2017;33(4):e00040717. http://dx.doi.org/10.1590/0102-311x00040717. PMid:28538787.

28. Bernardo WM, Jatene FB, Nobre MRC. Experiência clínica, educação médica continuada e qualidade da atenção em saúde. Rev Assoc Med Bras. 2005;51(2):63-4. http://dx.doi.org/10.1590/S0104-42302005000200004.

29. Davidoff F, Haynes B, Sackett D, Smith R. Evidence based medicine. BMJ: British Medical Journal. 1995;310(6987):10856. http://dx.doi.org/10.1136/bmj.310.6987.1085. PMid:7742666.

30. Ferreira MP, Pitta MT. Avaliação da eficiência técnica na utilização dos recursos do sistema único. São Paulo Perspect. 2008;22(2):55-71.

31. Mendes JDV, Bittar OJNV. Perspectivas e desafios da gestão pública no SUS. Revista da Faculdade de Ciências Médicas de Sorocaba. 2014;16(1):35-9.

32. Martins LF, Laport TJ, Menezes VdP, Medeiros PB, Ronzani TM. Esgotamento entre profissionais da Atenção Primária à Saúde. Ciênc saúde coletiva. 2014;19(12):4739-50.

\section{Contribuição dos autores}

Todos os autores participaram do planejamento e execução do trabalho. 